\title{
STRESS ET SATISFACTION AU TRAVAIL DES CADRES INTERMÉDIAIRES DANS LES HÔPITAUX DE SUISSE ROMANDE DANS UN CONTEXTE DE RÉFORMES
}

\section{David Giauque et al.}

De Boeck Supérieur | @GRH

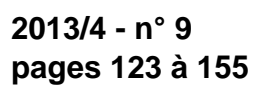

\section{ISSN 2034-9130}

Article disponible en ligne à l'adresse:

http://www.cairn.info/revue-@grh-2013-4-page-123.htm

Pour citer cet article :

Giauque David et al., " Stress et satisfaction au travail des cadres intermédiaires dans les hôpitaux de Suisse romande dans un contexte de réformes

",

@GRH, 2013/4 n 9, p. 123-155. DOI : 10.3917/grh.134.0123

Distribution électronique Cairn.info pour De Boeck Supérieur.

(c) De Boeck Supérieur. Tous droits réservés pour tous pays.

La reproduction ou représentation de cet article, notamment par photocopie, n'est autorisée que dans les limites des conditions générales d'utilisation du site ou, le cas échéant, des conditions générales de la licence souscrite par votre établissement. Toute autre reproduction ou représentation, en tout ou partie, sous quelque forme et de quelque manière que ce soit, est interdite sauf accord préalable et écrit de l'éditeur, en dehors des cas prévus par la législation en vigueur en France. II est précisé que son stockage dans une base de données est également interdit. 


\title{
STRESS ET SATISFACTION AU TRAVAIL DES CADRES INTERMÉDIAIRES DANS LES HÔPITAUX DE SUISSE ROMANDE DANS UN CONTEXTE DE RÉFORMES'
}

\author{
David Giauque \\ Professeur \\ david.giauque@unil.ch \\ Fabien Resenterra \\ Assistant-doctorant \\ fabien.resenterra@unil.ch

\section{Michaël Siggen} \\ Assistant-doctorant \\ michael.siggen@unil.ch
}

Tous trois travaillent à I'Université de Lausanne, Faculté des sciences sociales et politiques, Institut d'études politiques et internationales

Résumé

L'article vise à identifier les déterminants du stress et de la satisfaction au travail parmi une population de cadres intermédiaires exerçant dans des établissements hospitaliers de Suisse romande. II vise également à combler un vide dans les études sur les administrations publiques suisses : mieux saisir, au moyen d'une enquête par questionnaires, quelles sont les principales variables qui se trouvent à l'origine de la satisfaction au travail et qui peuvent être des ressources pour les acteurs afin de faire face au stress. L'étude confirme des résultats de recherches internationales, montrant que des facteurs relationnels et des caractéristiques du travail sont les principaux déterminants du stress et de la satisfaction. Elle souligne dès lors l'importance d'adopter une lecture "structurelle " et " organisationnelle " du stress et de la satisfaction, soulignant aussi que les caractéristiques individuelles de la population étudiée n'expliquent qu'une très infime variance de ces phénomènes.

[1] Cet article est basé sur les données d'un projet de recherche plus large financé par le Fonds National Suisse de la Recherche Scientifique, Division des sciences humaines et sociales, projet DORE - instrument de promotion pour la recherche orientée vers la pratique (projet no. 13DPD6_134764/1). 


\section{Mots-clés}

Stress ; satisfaction au travail ; cadres intermédiaires ; hôpitaux suisses ; enquête par questionnaire

Abstract

This article aims to identify antecedents of stress and job satisfaction among a population of middle-range managers working in public hospitals in the French part of Switzerland. It tries to fill in an information gap with respect to research on Swiss public administrations : to better identify, by means of a survey, the main dimensions which might explain job satisfaction, and which could be considered as resources to fight against stress situations. The study confirms previous international findings, showing that relational factors and work characteristics are the main explanatory variables of stress and job satisfaction. This research underlines the importance to consider primarily structural and organizational antecedents when studying stress and job satisfaction, demonstrating thus that individual characteristics of the studied population account for a very small part of stress' and job satisfaction's variance.

\section{Keywords}

Stress ; job satisfaction ; middle-range employees ; Swiss hospitals ; survey

\section{INTRODUCTION}

Les hôpitaux publics de Suisse romande se trouvent aujourd'hui confrontés à de nombreux défis d'envergure. Des réformes rapides, pour ne pas dire brutales, les touchent fortement, de sorte qu'ils sont amenés à entreprendre des changements organisationnels tant sur les plans stratégique, managérial, comptable/financier et en termes de gestion des ressources humaines. À ce jour, peu d'enquêtes empiriques ont porté sur les conséquences de ces différentes transformations managériales sur les acteurs des établissements hospitaliers suisses. De manière générale, les autorités publiques suisses se sont très peu intéressées aux questions touchant au stress professionnel et se trouvent donc peu informées par rapport à une telle problématique. Cette dernière touche, tout comme dans les autres pays de I'OCDE, bon nombre d'employés. Aussi, le présent article vise à combler une lacune en s'intéressant plus particulièrement au stress ressenti par les cadres intermédiaires d'établissements hospitaliers de Suisse romande, ainsi qu'à leur satisfaction. La question de recherche principale que nous abordons ici a trait aux origines de ce ressenti en matière de stress et de satisfaction chez les cadres intermédiaires (ou middle-range managers en anglais). Partant d'un point de 
vue " structurel ", nous posons en hypothèse que le stress perçu et la satisfaction au travail peuvent s'expliquer, en premier lieu, par des aspects relationnels et les caractéristiques du travail de nos répondants. Autrement dit, nous prenons des distances par rapport aux travaux " psychologisants ", expliquant le stress et la satisfaction par des variables individuelles, pour nous inscrire plutôt dans une perspective constructiviste du phénomène du stress, puisque nous postulons que des conditions de travail objectives ont des répercussions sur la perception subjective du stress et de la satisfaction des acteurs, celle-ci en retour pouvant affecter la réalité objective des conditions de travail. Cette communication est donc novatrice à plusieurs égards. Tout d'abord, elle s'intéresse à la question du stress perçu et de la satisfaction au travail dans les établissements hospitaliers de Suisse romande dans un contexte de réformes importantes du système de santé. Ce contexte particulier, marqué par de nombreuses initiatives politiques et managériales, n'est évidemment pas neutre quant aux perceptions en termes de stress et de satisfaction des acteurs du système de santé suisse. Deuxièmement, l'étude porte sur une population particulière des établissements hospitaliers : les cadres intermédiaires. Leur position à cheval entre leurs pratiques professionnelles d'une part, et la gestion de leur unité d'autre part, les met directement aux prises avec des logiques sectorielles différentes et souvent contradictoires. La première, venant " d'en haut ", vise la mise en œuvre des nouvelles directives et réformes au sein des unités de soins, afin d'en améliorer la performance économique (réorganisation des lits, regroupement de tâches, gestion des ressources humaines, etc.). La deuxième, venant " d'en bas ", vise la représentation et le respect des normes et pratiques professionnelles. Cette double injonction, faite à ces acteurs de l'interface, oblige ces derniers à redéfinir tant la représentation qu'ils se font de leur rôle au sein de l'organisation que leur identité professionnelle. Dès lors, cette situation d'interface rend cette population hospitalière particulièrement vulnérable à des problématiques de stress. Finalement, l'article vise à mieux comprendre les déterminants du stress et de la satisfaction de ces acteurs, en soulignant le rôle que peuvent jouer leurs conditions de travail et leurs relations sociales. En ce sens, il nous sera dès lors possible de pointer certains facteurs " organisationnels » et " relationnels » qui peuvent être causes de souffrance et de plaisir au travail. Afin d'atteindre ces différents objectifs et de répondre à notre question de recherche, l'article est structuré de la manière suivante. Dans un premier temps, pour que le lecteur puisse mieux appréhender les réformes hospitalières helvétiques, nous revenons sur les principales décisions politiques et managériales qui affectent le secteur hospitalier. Nous posons ensuite quelques éléments théoriques concernant les notions de stress et de satisfaction au travail afin de proposer des hypothèses de recherche qui nous permettent de guider nos réflexions empiriques subséquentes. Puis nous présentons notre méthodologie d'enquête, les données à partir desquelles les résultats sont tirés, les mesures que nous utilisons, de même que les traitements statistiques que nous 
réalisons. Nous exposons ensuite nos principaux résultats pour les discuter et en tirer les principales conclusions. Nous terminons par l'exposé des limites propres à la présente recherche.

\section{LE CONTEXTE : LES RÉFORMES HOSPITALIÈRES}

Lorsque la Loi fédérale sur l'assurance-maladie (ci-après LAMal) est introduite en 1996, deux buts principaux sont poursuivis : I'amélioration de la situation des assurés face au risque de la maladie et l'introduction d'instruments de maîtrise des coûts. À ces deux objectifs s'ajoute également celui du cadre juridique dans lequel s'insère la LAMal et qui agit également sur ces coûts. L'existence de 26 systèmes de santé semi-autonomes, du fait des prérogatives cantonales en matière de mise en œuvre de la politique publique hospitalière ${ }^{2}$, rend difficile la mise en place de marchés concurrentiels au niveau suisse et favorise la densité hospitalière, impliquant par conséquent des besoins en équipement particulièrement coûteux. Depuis le début des années 2000, le Conseil fédéral a soumis au Parlement diverses mesures afin de réformer la LAMal, lesquelles auront connu des destins divers. Nous nous concentrons ici sur la réforme du financement hospitalier adoptée en 2007, qui a d'importantes répercussions managériales au niveau des hôpitaux suisses.

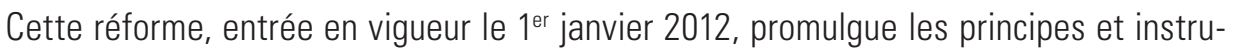
ments de gestion suivants :

D'un financement des coûts à un financement des prestations. Avec la réforme du financement hospitalier, les coûts d'exploitation et d'investissement sont financés conjointement, ce qui signifie que les montants nécessaires à la couverture des coûts d'exploitation (traitements médicaux et séjour à l'hôpital) ainsi que le financement des investissements sont compris dans les forfaits liés aux prestations. Les prestations d'intérêt général, comme la formation universitaire et la recherche, sont financées séparément sous la forme de mandats de prestations attribués par les cantons.

Harmonisation de la structure tarifaire. Dès 2012, le secteur hospitalier suisse est passé à une indemnisation selon les forfaits par cas, ou Diagnosis Related Groups (DRG), lesquels sont basés sur une structure tarifaire uniforme à toute la Suisse et dont la gestion a été confiée à SwissDRG SA ${ }^{3}$. Les tarifs appliqués sont calculés sur les coûts effectifs des hôpitaux suisses. Les hôpitaux se voient ainsi contraints de fournir des prestations dont les coûts correspondent à cette moyenne afin de ne pas engendrer de déficit.

\footnotetext{
[2] Pour rappel la Suisse comprend 26 cantons.

[3] Société anonyme composée de la Conférence suisse des directrices et des directeurs cantonaux de la santé (CDS), les Hôpitaux de Suisse $(\mathrm{H}+$ ), les assureurs-maladie suisses (santésuisse), la Fédération des médecins suisses (FMH), les assureurs selon la loi sur l'assurance-accidents, l'assurance-invalidité et l'assurance militaire.
} 
La révision du mode de financement hospitalier vise une harmonisation de la structure tarifaire sur le plan suisse devant aboutir, à terme, à une meilleure efficience dans la gestion des coûts hospitaliers. Cette nouvelle structure tarifaire est censée amener également plus de transparence concernant les coûts des prestations fournies tout en systématisant les comparaisons entre les pratiques des différents hôpitaux. En d'autres termes, cette révision a pour finalité d'inciter les acteurs du milieu hospitalier à fournir des prestations qui satisfassent aux critères d'économicité et de concurrence prévus par l'entrée en vigueur de la loi en 2012.

Les principales incidences de ces différentes réformes sur les hôpitaux sont les suivantes, sachant que nous manquons encore de recul pour en faire une liste exhaustive :

Concurrence. Les tarifs, ou prix des prestations, sont basés sur les coûts des hôpitaux (art. 49 al.1 LAMal) et les tarifs applicables sont eux basés sur les hôpitaux les plus efficients. En conséquence de quoi, les hôpitaux qui ne fournissent pas des prestations au même niveau de coûts que les hôpitaux les moins chers ne voient pas l'ensemble de leurs dépenses couvertes par les forfaits, induisant des déficits. La possibilité pour les assurés de choisir un hôpital hors de leur canton de résidence (libre circulation des patients) induit également une plus grande concurrence entre les hôpitaux.

Gestion par mandat de prestations : feuilles de route, tableau de bord et indicateurs de performance. Avec la réforme du financement hospitalier, les cantons ainsi que les assureurs n'indemnisent que les hôpitaux inscrits sur les listes, c'est-à-dire ceux pour lesquels les cantons ont attribué des mandats de prestations pour l'approvisionnement médical (art. 39 al.1 let.a de la LAMal et 58e de I'OAM). Au moyen de ces mandats de prestations, les cantons fournissent des fonds et fixent les prestations que les hôpitaux s'engagent à fournir, lesquelles sont suivies avec des indicateurs de performance.

Codage SwissDRG. La mise en place des forfaits par cas, sur la base de la structure tarifaire SwissDRG, nécessite un codage des prestations fournies aux patients. À partir d'un système de classification établi par l'Office fédéral de la statistique, l'ensemble des diagnostics et des procédures liés au traitement d'un patient est codé et classé, de son entrée à I'hôpital jusqu'à sa sortie.

Réorganisations médico-techniques. Des mesures complémentaires viennent s'ajouter à ces nouveaux outils de gestion. On retrouve notamment ce que les spécialistes appellent le " virage ambulatoire " et qui consiste à offrir des soins ponctuels en milieu hospitalier tout en maintenant, dans la mesure du possible, ces personnes dans leur milieu de vie habituel (HUG, Plan stratégique 2006-2010).

Réformes managériales. L'évolution des conditions cadres présentées ci-dessus ont donc poussé les autorités politiques cantonales à encourager leurs hôpitaux à revoir leurs méthodes de management, en particulier la conduite stratégique, l'organisation des structures et des processus, le contrôle de gestion ainsi que la gestion des 
ressources humaines. En matière de conduite stratégique, les directions hospitalières ont redéfini leurs missions, clarifié leur vision et fixé des axes stratégiques qui permettent de prioriser leurs projets ${ }^{4}$. Les dispositifs de contrôle de gestion sont considérablement renforcés : comptabilité analytique des prestations, indicateurs et tableaux de bord, études comparatives recourant au benchmarking interne et externe, permettant de suivre l'efficacité et l'efficience des prestations fournies par chaque département, et de les comparer entre différents établissements. Par ailleurs, à des fins d'économie, des coupes budgétaires sont réalisées, le manque de ressources apparaît dès lors plus clairement, d'autant que bon nombre d'hôpitaux suisses souffrent actuellement d'un sous-effectif pouvant être dû à des difficultés de recrutement, à l'absentéisme élevé des personnels de soin, ou à des politiques publiques visant à restreindre l'accès aux études de médecine (le numerus clausus). D'importants projets de réorganisation des structures et des processus, qui s'inspirent des méthodes de qualité totale, visent à optimiser les synergies entre départements, la transversalité et l'échange entre les corps professionnels (particulièrement la collaboration soignants-médecins) et plus généralement à améliorer l'efficience des processus de travail. Ces méthodes introduisent des bouleversements dans les habitudes de fonctionnement (Staines, 2000, 2007), et leurs conséquences sur le personnel ne sont souvent pas anticipées (Dahouadi et al., 2008 ; Pesqueux, 2008). En effet, de telles pratiques ont tendance à exacerber le sentiment de concurrence interne (Bolgiani, 2002), voire une forme de pensée comptable nuisible à la collaboration (Hufty, 1998). Elles peuvent de plus introduire une perception " clientéliste » des patients (Maugeri, 2006), impropre à une médecine de qualité et contraire à l'idée de " bon travail " qu'ont les personnels de la base. Finalement, elles sont souvent perçues comme une surcharge administrative qui éloigne le personnel soignant des patients, renforçant la tendance à la bureaucratisation du travail des employés publics. Une dernière catégorie de réformes managériales relève de la gestion des ressources humaines. Des changements considérables ont été introduits dans l'ensemble des fonctions publiques (Demmke et Moilanen, 2010 ; Emery et Giauque, 2007), et les hôpitaux publics ont naturellement emboîté le pas. De manière générale, les hôpitaux suisses, et notamment ceux qui ont été l'objet de nos investigations dans la présente étude, sont gérés aujourd'hui selon les principes et outils propres au mouvement de la nouvelle gestion publique (ou NGP) (Varone et de Visscher, 2004).

\section{CADRE THÉORIQUE ET HYPOTHĖSES DE RECHERCHE}

Eu égard aux changements importants auxquels les hôpitaux suisses sont actuellement confrontés et étant donné les profondes mutations managériales que ceux-ci induisent,

[4] Voir par ex. pour le CHUV : http://www.chuv.ch/le-chuv-en-bref/missions-chuv.htm ou http://www.hug-ge.ch/ hug_enbref/plan_strategique.html, pour les HUG (consultés le 2402 2010). 
il est intéressant de nous pencher sur les facteurs qui peuvent être en lien avec le stress et la satisfaction au travail parmi le personnel hospitalier. Plus précisément, notre recherche se focalise sur une population hospitalière spécifique : les cadres intermédiaires. Les raisons pour lesquelles cette étude porte sur cette dernière population sont multiples : la première à trait au fait que les cadres intermédiaires sont bien souvent ignorés dans les enquêtes sur la santé au travail en Suisse, une population souvent invisible ; la seconde est en lien avec les activités spécifiques des cadres intermédiaires qui sont à la fois proches du " terrain ", en contact avec les " clients et/ou patients ", tout en réalisant des tâches " d'encadrants ", devant bien souvent relayer et mettre en œuvre les directives provenant du management. La littérature sur les cadres intermédiaires évoque du reste fort bien les tensions/conflits de rôle auxquels ils peuvent être soumis. Or, les conflits de rôle sont particulièrement en lien avec le stress comme l'ont démontré des écrits précédents (Kahn et al., 1964). Ils constituent donc une population particulièrement adaptée à notre étude qui vise à comprendre quels sont les antécédents organisationnels et relationnels du stress et de la satisfaction au travail dans un contexte de réformes managériales particulièrement important. Ce d'autant plus que les hôpitaux constituent des univers " professionnels " spécifiques (Ferlie et al., 2001), des organisations hautement professionnalisées (Ferlie et al., 1996), ou des bureaucraties professionnelles selon la terminologie employée par Mintzberg (1982). Les réformes mettent clairement les normes professionnelles en souffrance, diminuant parfois de manière importante les marges de manœuvre des professionnels qui sont soumis à des injonctions managériales et économiques souvent fortement éloignées des valeurs qui fondent leur identité professionnelle (Evetts, 2009, 2013). Pour toutes les raisons évoquées ci-dessus, les cadres intermédiaires, en tant que dépositaires des normes professionnelles et intermédiaires des réformes managériales contemporaines, constituent une population intéressante lorsqu'il s'agit de comprendre quels sont les antécédents du stress et de la satisfaction au travail dans les hôpitaux suisses.

Dans la suite de cette section, nous revenons sur les principaux acquis théoriques et empiriques en lien avec le stress et la satisfaction au travail, ceci afin d'identifier les variables qui sont considérées comme explicatives de ces différentes attitudes au travail. Soulignons d'emblée qu'il existe un intérêt intellectuel à prendre en considération à la fois le stress et la satisfaction au travail, dans la mesure où des études ont montré que ces deux variables sont des facteurs explicatifs importants particulièrement dans un contexte de réformes organisationnelles (Noblet et Rodwell, 2009 ; Yousef, 2000). Partant de ces constats, nous souhaitons mettre en évidence les facteurs organisationnels contribuant au développement du sentiment de stress, mais également de satisfaction au travail parmi un échantillon de cadres intermédiaires évoluant en milieu hospitalier. 


\section{, 3.1. Le stress}

Afin de construire un modèle intellectuel pour appréhender le stress, il est important de revenir sur les modèles théoriques à disposition qui isolent certains antécédents. La plupart de ces modèles sont utilisés afin de mesurer le degré de stress ou de mal-être chez les salariés. Autrement dit, ces modèles sont principalement utilisés dans le cadre d'enquêtes de type quantitatif. Les deux principaux modèles, du moins les plus répandus, sont ceux de Karasek et de Siegrist. Le premier modèle comprend deux dimensions principales de l'organisation du travail. D'une part, la charge de travail du salarié, plus spécifiquement la charge psychologique attachée aux pressions temporelles (demand) et, d'autre part, la marge de manœuvre dont le salarié peut bénéficier ou pas dans ses activités professionnelles (control) (Bakker et al., 2008 ; Karasek, 1979 ; Noblet et al., 2006). Ce modèle prédit qu'une forte charge psychologique est associée à un niveau élevé de stress perçu et éventuellement à une prévalence plus élevée de souffrance psychique. Une forte exigence de travail sera moins préjudiciable, voire aucunement, si la personne concernée garde une marge de manœuvre importante dans son travail, la possibilité de participer à la définition des règles qui s'y rapportent, ainsi que de pouvoir faire largement usage de ses compétences professionnelles et sociales. Ce modèle "charge psychologique-marge de manœuvre " de Karasek s'est vu enrichir d'une troisième dimension par Johnson : le soutien social (Johnson, 1986 ; Johnson et Hall, 1988). Celui-ci comprend trois dimensions : le soutien hiérarchique (directeur, superviseur), celui des collègues (soutien collégial) et celui de la famille et des amis (soutien extra-professionnel). Le soutien social diminue l'impact négatif de la surcharge de travail lorsque celle-ci prévaut (Papart, 2002, 2007). Siegrist a, quant à lui, développé un autre modèle puisqu'il identifie un rapport de modulation réciproque entre la charge psychologique et la reconnaissance sociale au sein de l'organisation : le modèle " effort-reward imbalance at work ». L'effort de travail est d'autant plus générateur de stress que le salarié n'est pas, subjectivement ou objectivement, suffisamment récompensé ou reconnu pour ses efforts. Pour favoriser l'estime de soi du travailleur, un équilibre doit exister entre l'effort qu'il fournit et la reconnaissance qu'il en retire - " effort-reward model ». Autrement dit, un travail trop exigeant nuit à la santé quand sa reconnaissance matérielle et immatérielle n'est pas en rapport avec les efforts consentis par le salarié (Siegrist, 1996 ; Siegrist et al., 1990 ; Siegrist et al., 1986), tout en péjorant sa perception de la justice organisationnelle (Thelen, 2007). Les modèles développés par Karasek et par Johnson ont fait l'objet de plusieurs recherches empiriques quantitatives qui ont permis de montrer que les facteurs ainsi isolés, à savoir la charge, la marge de manœuvre, le soutien social, sont effectivement des éléments explicatifs majeurs quant au mal-être des salariés.

Un autre modèle, nommé " JD-R " (pour Job demands-resources model) (Bakker et Demerouti, 2007 ; Demerouti et al., 2001), a également fait l'objet de nombreuses études dans différents contextes de travail et est considéré comme empiriquement 
fondé. Celui-ci est, bien entendu, construit sur la base des théories précédemment présentées démontrant que le stress et le mal-être au travail résultent de deux phénomènes qui se trouvent en interaction : les contraintes spécifiques à l'organisation et au travail auxquelles les salariés sont confrontés, mais également le degré de contrôle que ces derniers peuvent déployer pour faire face à leurs impératifs et défis professionnels. Les développements théoriques et empiriques propres à ce JD-R modèle peuvent ainsi être considérés comme une tentative de synthèse des autres approches développées auparavant. De sorte qu'une masse considérable de recherches ont été réalisées en référence à ce modèle ces dernières années (Maslach et al., 1996 ; Schaufeli et Enzmann, 1998 ; Schaufeli et Bakker, 2004). Par ailleurs, ce modèle JD-R identifie une variété importante d'aspects du travail qui affectent le bien-être des salariés. Ces aspects peuvent être classés en deux catégories opposées : les exigences portant sur les salariés et les ressources dont ils peuvent disposer pour y faire face. D'une part, donc, " job demands are defined as those aspects of the work context that tax employees' personal capacities and are, therefore, associated with certain psychological and/or physiological costs " (Van den Broeck et al., 2008 : 278). La surcharge de travail, les contraintes organisationnelles et procédurales, les demandes émotionnelles, les conflits entre vie privée et vie professionnelle ainsi que les conflits interpersonnels sont des facteurs qui ont été identifiés comme étant positivement associés au stress des salariés si ceux-ci dépassent leurs capacités à y faire face (Bakker et al., 2008 ; Noblet et al., 2006 ; Richardsen et al., 1992 ; Schaufeli et Bakker, 2004). D'autre part, " the category of job resources is defined as those physical, psychological, social, or organizational aspects of the work context that (1) can reduce the health impairing impact of job demands, (2) are functional in achieving work goals, and (3) stimulate personal growth, development, and learning " (Van den Broeck et al., 2008 : 278). Les opportunités liées à l'utilisation de ses qualifications et compétences professionnelles, le soutien des supérieurs hiérarchiques et des collègues, les récompenses financières, les opportunités de promotion et de carrière, l'ambiance de travail au sein d'une équipe, l'autonomie dans le travail sont autant de facteurs qui ont été identifiés comme pouvant constituer des ressources importantes pour les salariés (Bakker et Demerouti, 2007 ; Demerouti et al., 2001 ; Johnson et Hall, 1988 ; Schaufeli et Bakker, 2004 ; Siegrist, 1996). Ainsi, la plupart du temps, les dimensions qui ont été considérées comme pouvant affecter positivement le stress professionnel (des ressources autrement dit) sont : sociales (le soutien des supérieurs hiérarchiques et des collègues) ou liées au travail et/ou à l'organisation (l'autonomie et les responsabilités ; les opportunités de développement ; les récompenses tangibles ou intangibles ; la possibilité de pouvoir utiliser ses compétences ou qualifications). Dans le cadre de notre recherche, nous avons également souhaité considérer d'autres aspects organisationnels pouvant avoir également un effet sur le stress perçu par les salariés, notamment du fait que les répondants à notre enquête représentent une population particulière, c'est-à-dire des " middle-range 
managers ». Aussi, ces derniers jouent bien souvent un rôle de relais informationnel, et une ressource importante pour eux dans le cadre de la réalisation de leurs activités professionnelles se situe au niveau de la qualité des flux d'information et de communication. De même, s'ils sont des relais informationnels, ils jouent aussi le rôle de prescripteurs du travail de leurs subordonnés en leur demandant, notamment, de se conformer à certaines règles, procédures et contraintes administratives. Celles-ci peuvent donc constituer des ressources, mais peuvent également se transformer en contraintes importantes si elles deviennent envahissantes et si la formalisation du travail est trop poussée ${ }^{5}$ (Bozeman, 2000 ; Brewer et Walker, 2010 ; Giauque, Anderfuhren-Biget et al., 2013 ; Giauque, Ritz, et al., 2012 ; Pandey et Scott, 2002).

\subsection{La satisfaction au travail}

Comme l'indique la littérature spécialisée, il existe un intérêt intellectuel de contrebalancer la recherche des antécédents du stress par une réflexion sur les facteurs menant à la satisfaction au travail (Noblet et Rodwell, 2009 ; Yousef, 2000). Nous mobilisons ainsi ce concept de satisfaction au travail principalement pour rendre compte des déterminants permettant le développement du plaisir au travail. Comme l'indique la littérature sur la santé au travail, plaisir et souffrance sont bien souvent les deux faces d'une même réalité et mérite donc une enquête simultanée (Carpentier-Roy, 1995 ; Dejours, 1987). Par ailleurs, l'étude simultanée du stress et de la satisfaction au travail peut permettre une comparaison intéressante de leurs antécédents respectifs et ouvrir des pistes intéressantes en termes d'analyse.

En regard de la littérature y relative, le concept de satisfaction au travail concerne principalement les réponses des individus à l'égard de leur expérience professionnelle. II s'agit probablement de l'attitude à l'égard du travail la plus étudiée. Une grande partie des scientifiques soulignent que la satisfaction au travail mesure en fait l'attitude des salariés en réponse à leurs expériences de travail (Mowday et al., 1982). Selon Taylor, " most efforts to explain job satisfaction are based on the person-environment fit paradigm. Put simply, workers with higher satisfaction levels are those who have developed a better fit psychologically between their personal needs or values and the characteristics of their jobs. (...) Employees who are satisfied with their jobs will perceive that their job fulfils, or allows for the fulfillment of, values that are important to them " (2007: 939). Autrement dit, la satisfaction au travail est largement définie, dans la littérature, comme étant un état émotionnel positif résultant de l'évaluation réalisée par les salariés à l'égard de leur emploi (Agho et al., 1993 ; Locke, 1976).

Bien que l'intérêt pour ce concept de satisfaction au travail soit actuellement remis en question et considéré par certains comme " a throw-away variable » (Staw, 1984),

[5] À cet égard, voir les travaux en lien avec la notion de Red Tape largement développée dans la littérature anglosaxonne. 
d'autres critiques soulignent que le construit de satisfaction au travail a finalement reçu peu d'attention malgré des apparences trompeuses (Büssing, 1992). D'autres écrits appellent à la prudence dans I'utilisation de cette notion, remarquant au passage la constante proportion d'employés déclarant des hauts niveaux de satisfaction dans les entreprises, rendant dès lors cette notion suspecte à leurs yeux (Szilagyi et Wallace, 1983 ; Weaver, 1980). Quoi qu'il en soit, le concept de satisfaction au travail a été défini de façons fort différentes dans de nombreux textes. En conséquence, sa mesure constitue toujours une controverse académique, et il existe de nombreuses manières d'évaluer la satisfaction des salariés, mais nous ne traiterons pas de cette question spécifique dans le présent article.

Plusieurs théories portant sur les antécédents de la satisfaction au travail ont été développées. Celles-ci peuvent être rapidement classées en trois catégories :

- Les théories " situationnelles ", qui partent de l'hypothèse selon laquelle la satisfaction au travail est principalement la résultante de la nature du travail ou d'autres aspects de l'environnement du travail.

- Les théories "dispositionnelles", quant à elles, stipulent que la satisfaction au travail trouve ses origines dans les méandres psychologiques des individus.

- Finalement, les théories "interactionnistes", qui soulignent que la satisfaction au travail est le produit d'une interaction entre un contexte particulier et des facteurs plus psychologiques et individuels (Judge et Klinger, 2007 : 398-399).

En rapport avec les théories dites "situationnelles", que nous prendrons plus particulièrement en considération dans cet article, plusieurs auteurs démontrent que les activités contenant des caractéristiques motivationnelles intrinsèques vont contribuer à un plus haut niveau de satisfaction au travail que les emplois dont les caractéristiques motivationnelles sont principalement liées à des aspects extrinsèques (Cohen, 2012 ; Frey, 1997). Cinq caractéristiques peuvent être considérées ainsi comme faisant partie d'un travail intrinsèquement motivant : (1) l'identité de la tâche ou la manière dont le salarié peut conduire son travail du début à la fin ; (2) la signification de la tâche ou la façon dont un salarié considère sa tâche en termes d'importance et de signification ; (3) la variété des tâches ou la possibilité donnée à un salarié d'utiliser ses qualifications et compétences dans ses activités ; (4) l'autonomie ou le fait de pouvoir bénéficier d'une marge de manœuvre, de discrétion (d'un contrôle) sur les tâches professionnelles ; finalement, (5) le feedback ou la possibilité offerte aux salariés d'avoir un retour sur leurs activités, notamment sur la manière dont ils réalisent leur travail (Judge et Klinger, 2007 : 399). De nombreuses études empiriques ont souligné l'intérêt d'un tel point de vue, montrant que quand les employés sont amenés à évaluer différents aspects de leur travail (la supervision, la rémunération, les opportunités de promotion, la collaboration, etc.), bien souvent le contenu du travail lui-même est considéré comme la dimension la plus importante (Judge \& Church, 2000). Des résultats similaires peuvent être identifiés dans la 
littérature portant sur les administrations publiques. Ainsi, se basant sur des données secondaires portant sur plus de 14000 salariés publics néerlandais, Steijn (2004) a pu démontrer que l'impact des caractéristiques individuelles sur la satisfaction au travail est quasiment nul, alors même que les caractéristiques organisationnelles et du travail peuvent être considérées comme les variables explicatives les plus importantes de la satisfaction de cette population. Dans une même optique, Noblet et Rodwell (2009) soulignent que les officiers de police australiens sont susceptibles de déclarer un plus haut niveau de satisfaction au travail s'ils peuvent bénéficier de latitudes décisionnelles, d'une indépendance au travail, mais également du soutien de la hiérarchie et des collègues. Les caractéristiques propres au travail (l'autonomie et de bonnes relations de travail avec les supérieurs et les collègues notamment) sont également identifiées comme les antécédents les plus importants de la satisfaction au travail dans d'autres études comparatives incluant des agents publics de sept pays différents (Taylor et Westover, 2011). Les résultats précédents indiquent donc que la satisfaction au travail est plus particulièrement associée à des caractéristiques du travail, son contenu, ainsi qu'à la qualité des relations sociales (soutien des collègues et des supérieurs hiérarchiques).

\section{, 3.3. Hypothèses de travail ou attentes théoriques}

Sur la base de ces considérations théoriques liées aux principaux facteurs explicatifs du stress et de la satisfaction au travail, nous avons identifié plusieurs variables que nous utilisons dans notre propre enquête. Ainsi, deux variables relationnelles sont prises en considération (relations avec les collègues et les supérieurs hiérarchiques). Comme nous l'expliquions auparavant ces variables se sont révélées très utiles, dans des recherches antérieures, pour expliquer le stress perçu et la satisfaction au travail. D'autre part, un choix de variables en lien avec le fonctionnement organisationnel ont pu être identifiées dans la littérature comme des antécédents du stress et de la satisfaction. II s'agit des variables suivantes : une marge de manœuvre dans l'organisation du travail ; l'autonomie, l'implication et la responsabilité personnelles dans les prises de décision ; les possibilités de développement offertes dans le cadre du travail ; les contraintes bureaucratiques ou formelles (les « red tapes " que nous évoquions plus avant dans notre article); ainsi que les récompenses tangibles (dans notre cas nous considérons ici principalement la rémunération). Nous complétons cette batterie de variables en incluant également l'information et la communication au sein des organisations comme étant potentiellement un facteur explicatif du stress et de la satisfaction au travail, notamment du fait des nombreuses recherches ayant conduit à considérer les flux d'information comme centraux dans les processus de coordination et coopération au sein des organisations (Feldman et March, 1991). Dès lors, le tableau suivant récapitule les relations hypothétiques entre nos variables indépendantes, ci-dessus relevées, et nos variables dépendantes (stress et satisfaction au travail). 


\section{Tableau 1. Attentes théoriques entre nos variables indépendantes et nos variables dépendantes}

\begin{tabular}{|l|c|c|}
\hline & Stress perçu & Satisfaction au travail \\
\hline Relations avec les collègues & - & + \\
\hline Relations avec les supérieurs & - & + \\
\hline Organisation du travail & - & + \\
\hline Autonomie et implication dans la prise de décision & - & + \\
\hline Possibilités de développement personnel & - & + \\
\hline Contraintes bureaucratiques, règles formalisées & + & - \\
\hline Information et communication & - & + \\
\hline Rémunération & - & + \\
\hline
\end{tabular}

- = soulignant une association négative avec la variable dépendante

$+=$ soulignant une association positive avec la variable dépendante

\section{MÉTHODES}

\section{, 4.1. Échantillon et procédure}

Les données de notre recherche ont été récoltées dans le cadre d'une recherche scientifique portant sur les cadres intermédiaires des établissements hospitaliers de Suisse romande. À l'origine, tous les cantons romands ont été sollicités afin de participer à l'enquête. Pour des raisons politiques, principalement, certains d'entre eux n'ont pas souhaité collaborer. Par contre, nous avons pu compter sur la participation de plusieurs établissements hospitaliers se situant dans les cantons de Neuchâtel, de Vaud, du Jura, et de Berne (partie francophone). Notre questionnaire, sous forme électronique, fut envoyé à un répondant au sein de ces établissements hospitaliers (généralement un responsable du service des ressources humaines), qui a ensuite envoyé le lien URL de notre questionnaire électronique au personnel cadre de son organisation. Cette manière de procéder était évidemment la plus simple pour l'équipe de recherche dans la mesure où nous n'avions pas besoin de gérer nous-mêmes les adresses électroniques de notre population-cible. Par ailleurs, soulignons que pour certains établissements, l'équipe de recherche a négocié une convention éthique (en collaboration avec un comité d'éthique cantonal) afin de pouvoir mener à bien l'enquête. Notons aussi que les données récoltées dans le cadre de nos questionnaires électroniques ont été stockées directement sur un serveur de l'une de nos institutions universitaires pour éviter ainsi tout problème de confidentialité. Les participants à notre enquête ont été dûment informés de cette procédure et des conditions de confidentialité strictes que nous leur garantissions. Notre enquête a débuté le 24 janvier 2012 pour se terminer le 9 mai de la même année. Plusieurs rappels ont été envoyés à notre population-cible afin d'augmenter la participation des cadres intermédiaires et s'assurer ainsi une bonne représentativité de nos données. À cet égard, signalons que les taux de participation dans les différents 
établissements avoisinent les $70 \%$ mais que certains questionnaires, comme toujours dans ce type d'enquête, ne sont pas complètement remplis. Aussi, si nous avons pu réunir en tout 942 questionnaires, toutefois le nombre d'entre eux réellement exploitable est moindre puisque certaines parties du questionnaire comprenaient des non-réponses. Au final, nous avons pu exploiter 755 questionnaires complets.

Concernant les caractéristiques " démographiques » de notre échantillon, le tableau cidessous regroupe les principales informations à notre disposition.

Tableau 2. Caractéristiques de notre échantillon

\begin{tabular}{|c|c|}
\hline Variables: & Caractéristiques de la population \\
\hline Sexe : & $\begin{array}{l}45 \% \text { de femmes } \\
55 \% \text { d'hommes }\end{array}$ \\
\hline Âge par catégories : & $\begin{array}{l}20 \text { ans et moins : } 0.1 \% \\
21-30 \text { ans : } 1.5 \% \\
31-40 \text { ans : } 17.7 \% \\
41-50 \text { ans : } 39 \% \\
51-60 \text { ans : } 36 \% \\
61 \text { et plus : } 5.7 \%\end{array}$ \\
\hline Fonction par catégories : & $\begin{array}{l}\text { Autres cadres : } 32.4 \% \\
\text { Cadres administratifs : } 9.9 \% \\
\text { Cadres infirmiers : } 33.7 \% \\
\text { Cadres médecins : } 24 \%\end{array}$ \\
\hline Ancienneté dans la profession : & $\begin{array}{l}\text { Moins d'un an : } 2.1 \% \\
1-2 \text { ans : } 2.9 \% \\
3-5 \text { ans }: 6.1 \% \\
6-10 \text { ans : } 9.8 \% \\
11-15 \text { ans }: 17.6 \% \\
16-24 \text { ans : } 32.4 \% \\
25 \text { ans et plus : } 29.2 \%\end{array}$ \\
\hline Ancienneté dans la fonction: & $\begin{array}{l}\text { Moins d'un an : } 3.3 \% \\
1-2 \text { ans : } 7.8 \% \\
3-5 \text { ans }: 17.9 \% \\
6-10 \text { ans : } 20.2 \% \\
11-15 \text { ans : } 23.1 \% \\
16-24 \text { ans : } 20.6 \% \\
25 \text { ans et plus : } 7.1 \%\end{array}$ \\
\hline Ancienneté organisationnelle : & $\begin{array}{l}\text { Moins d'un an : } 1.4 \% \\
1-2 \text { ans : } 2.1 \% \\
3-5 \text { ans : } 5.5 \% \\
6-10 \text { ans : } 13.9 \% \\
11-15 \text { ans : } 19.2 \% \\
16-24 \text { ans }: 31.3 \% \\
25 \text { ans et plus : } 26.5 \%\end{array}$ \\
\hline
\end{tabular}




\section{, 4.2. Instruments}

Les différentes données utilisées dans le cadre de notre enquête ont fait l'objet de plusieurs traitements statistiques afin de s'assurer de leurs bonnes conditions. Aussi, elles ont été analysées afin de vérifier l'hypothèse de " normalité ". En regard des résultats, toutes les variables utilisées se trouvent dans des fourchettes statistiquement acceptables (le skewness devant être inférieur à 2 et le kurtosis inférieur à 7). Soulignons également que nos variables ont été construites après des analyses factorielles exploratoires (analyse en composante principale, rotation varimax), cette procédure permettant de vérifier si nos variables ne représentent qu'une seule composante. Suite de quoi la fiabilité de nos échelles de mesure fut vérifiée au moyen de l'Alpha de Cronbach. L'annexe 1 renseigne sur les différentes échelles de mesure utilisées dans le présent article.

\section{Les variables dépendantes}

Le stress perçu. Pour les fins de notre étude, nous avons utilisé la mesure du stress perçu développée par Cohen et al. (Cohen et al., 1983). Le grand avantage de l'utilisation de cette échelle de mesure est que nous bénéficions d'une version française qui a déjà fait l'objet d'une validation statistique (Bruchon-Schweitzer et Dantzer, 1994). Après I'analyse factorielle, nous avons retenu 6 items pour mesurer cette variable. L'Alpha de Cronbach de cette échelle est de .817. Au fin des régressions statistiques la moyenne des différents items composant l'échelle est utilisée (nous avons procédé de la même façon pour toutes les autres variables de cette étude).

La satisfaction au travail. Dans cette étude, la satisfaction au travail est mesurée via un seul item visant à rendre compte de la perception globale de la satisfaction de nos répondants. Nous sommes bien conscients qu'il est en général préférable de mesurer ce type d'attitude au travail au moyen de plusieurs items. Cependant, plusieurs études ont adopté une stratégie identique à la nôtre et certains auteurs estiment même qu'une telle mesure portant sur un item peut être considérée comme hautement fiable (Taylor, 2008). Quoi qu'il en soit, le fait qu'un seul item soit ici utilisé doit être relevé comme une limitation potentielle de la présente étude. Cet item est libellé de la manière suivante : " comment évaluez-vous votre satisfaction professionnelle actuelle ? " Cet item est mesuré au moyen d'une échelle de Likert à 10 points ( $1=$ très mauvaise ; $10=$ très bonne).

Les variables indépendantes développées dans l'étude, de même que leurs items se trouvent à l'annexe 1 du présent travail.

\section{Variables de contrôle}

Des variables de contrôle sont également utilisées dans nos divers modèles de régression présentés par la suite. Ces variables visent à contrôler si des caractéristiques " individuelles " peuvent expliquer une proportion de la variance de nos deux variables dépendantes. Ces variables de contrôle sont : le sexe ; l'âge par catégories ; la fonction occupée selon des catégories ; I'ancienneté dans la profession ; l'ancienneté dans la fonction de cadre ; et finalement l'ancienneté organisationnelle. 


\subsection{Analyse}

Pour le compte de cette étude particulière, nous avons procédé à la réalisation de deux régressions linéaires en deux étapes. La première de ces régressions linéaires concerne le stress perçu (variable dépendante). La première étape a consisté à introduire les variables de contrôle dans l'équation afin de pouvoir évaluer leur pouvoir explicatif unique. Dans une deuxième étape, nous avons ajouté dans l'équation les différentes variables concernant les caractéristiques du travail et les aspects relationnels. Cette manière de procéder nous permet de voir si des variables de contrôle sont toujours significativement corrélées avec le stress perçu en tenant compte des autres variables indépendantes. De plus, cette stratégie nous permet de comparer également le pouvoir explicatif de ces dernières variables comparativement aux variables de contrôle en mesurant la différence de $R^{2}$, c'est-à-dire la part de variance de notre variable dépendante expliquée d'une part par les variables de contrôle et, d'autre part, par nos variables mesurant les caractéristiques du travail et les facteurs relationnels. La méthode de sélection des variables dans la régression utilisée dans notre recherche est " entry block ", c'est-à-dire que toutes les variables sont entrées dans une seule et même opération mais en deux étapes, la première consistant à intégrer la totalité des variables de contrôle et la seconde visant à y ajouter les variables en relation avec les caractéristiques du travail.

Une stratégie comparable a également été utilisée pour mesurer les antécédents de la satisfaction au travail. Notons, finalement, que nous avons pris soin de vérifier les statistiques de colinéarité dans le cadre de nos différentes équations. La tolérance et le VIF constituent deux indicateurs importants. Pour nos différents modèles de régression, toutes les valeurs obtenues se trouvent dans les limites acceptables (tolérance > 3 ; VIF <3) (Carricano \& Poujol, 2008). Le test statistique dit Durbin-Watson a également été systématiquement effectué (test visant à s'assurer que les résidus dans le cadre d'une régression sont indépendants, écartant ainsi le risque d'autocorrélation) et nos résultats nous conduisent à considérer que nos régressions sont conformes aux fourchettes statistiques prescrites dans la littérature spécialisée.

\section{RÉSULTATS}

Le tableau 3, ci-dessous, fait état des résultats de notre première régression linéaire à deux étapes ayant le stress perçu comme variable dépendante. Un premier constat s'impose d'emblée. Aucune de nos variables de contrôle n'est significativement associée au stress. Autrement dit, les caractéristiques individuelles que nous avons sélectionnées dans notre équation ne sont pas corrélées significativement avec la variable "stress ". Par ailleurs, la part de variance du stress expliquée par ces variables de contrôle n'est que de $1.3 \%\left(R^{2}=.013\right)$, autrement dit négligeable. Par contre, une fois que les variables concernant les caractéristiques du travail sont introduites dans 
l'équation, on peut remarquer que la part de variance du stress expliquée par toutes nos variables atteint $32.9 \%$ (31.6 \% environ étant attribuable aux seules variables des caractéristiques du travail et des facteurs relationnels). En conséquence, un peu moins du tiers de la variance du stress perçu est ainsi expliqué par les variables figurant dans notre équation ce qui n'est guère, statistiquement parlant, anodin.

Tableau 3. Régressions linéaires concernant le stress perçu

\begin{tabular}{|c|c|c|c|}
\hline & & $\begin{array}{l}\text { Modèle } 1 \\
\text { Bêta (Signification) }\end{array}$ & $\begin{array}{l}\text { Modèle } 2 \\
\text { Bêta (Signification) }\end{array}$ \\
\hline $\begin{array}{l}\text { Variables } \\
\text { de contrôle : }\end{array}$ & $\begin{array}{l}\text { Sexe } \\
\text { Âge par catégories } \\
\text { Ancienneté professionnelle } \\
\text { Ancienneté fonctionnelle } \\
\text { Ancienneté organisationnelle } \\
\text { Cadres infirmiers } \\
\text { Cadres administratifs } \\
\text { Cadres médecins } \\
\text { Autres cadres }\end{array}$ & \begin{tabular}{|l}
$.008(.831)$ \\
$-.081(.111)$ \\
$-.008(.866)$ \\
$-.051(.334)$ \\
$.070(.155)$ \\
$-.019(.761)$ \\
$.023(.644)$ \\
$.053(.372)$ \\
$.010(.855)$ \\
\end{tabular} & $\begin{array}{l}-.006(.842) \\
-.057(.182) \\
-.031(.431) \\
.009(.835) \\
.054(.190) \\
-.033(.534) \\
.031(.454) \\
-.092(.085) \\
.027(.566)\end{array}$ \\
\hline $\begin{array}{l}\text { Variables } \\
\text { indépendantes : }\end{array}$ & $\begin{array}{l}\text { Rel_sup } \\
\text { Rel_col } \\
\text { Org_pers_trav } \\
\text { Com_inf_org } \\
\text { Auto_resp } \\
\text { Regl_proc } \\
\text { Dev_pers } \\
\text { Remuneration }\end{array}$ & & $\begin{array}{l}-.048(257) \\
-.162(.000)^{* * *} \\
-.310(.000)^{* * *} \\
-.067(.105) \\
-.117(.009)^{* *} \\
-.077(.043)^{*} \\
.018(.686) \\
-.033(.373)\end{array}$ \\
\hline $\begin{array}{l}\text { Récapitulatif } \\
\text { des modèles : }\end{array}$ & $\begin{array}{l}\mathrm{R}^{2}: \\
\text { Variation de } \mathrm{R}^{2} \text { : } \\
\mathrm{R}^{2} \text { ajusté : } \\
\text { Statistique F: } \\
\text { Test Durbin-Watson: }\end{array}$ & $\begin{array}{l}.013 \\
.013 \\
.001 \\
1.109(.353)\end{array}$ & \begin{tabular}{|l|}
.329 \\
.316 \\
.313 \\
$43.323(.000)^{* * *}$ \\
2.014
\end{tabular} \\
\hline
\end{tabular}

Parmi les variables indépendantes, les caractéristiques du travail et aspects relationnels suivants peuvent contribuer à diminuer le stress perçu par nos répondants. Prenons-les par ordre d'importance statistique. Tout d'abord, l'organisation personnelle du travail $(r=-.310 ; p<.001)$, puis l'état des relations avec les collègues $(r=-.162 ; p<.001)$, ou encore l'autonomie et les responsabilités $(r=-.117 ; p<.01)$. Finalement, de façon plus étonnante cette fois-ci, les règles, procédures et contraintes administratives $(r=-.077 ; p<.05)$ sont des facteurs qui, s'ils sont perçus comme des aspects satisfaisants du travail par nos répondants, sont négativement et significativement associés au stress perçu. Étonnamment, les relations avec les supérieurs hiérarchiques, la communication et les flux d'information au sein de l'organisation ainsi que les possibilités de 
développement personnel ne sont pas des aspects associés significativement au stress perçu. La rémunération, de façon beaucoup moins surprenante, ne l'est pas également. Si l'on s'intéresse maintenant à la satisfaction au travail de nos répondants, on peut tout d'abord constater qu'aucune variable " individuelle » n'est significativement corrélée avec cette attitude positive à l'égard du travail (modèle 1 du tableau 4 ci-dessous). Ici également les caractéristiques individuelles ne sont pas vraiment discriminantes pour expliquer la satisfaction au travail puisque les variables de contrôle n'expliquent que $2.8 \%$ de la variance de notre variable dépendante.

Tableau 4. Régressions linéaires concernant la satisfaction au travail

\begin{tabular}{|c|c|c|c|}
\hline & & \begin{tabular}{|l|} 
Modèle 1 \\
Bêta (Signification)
\end{tabular} & $\begin{array}{l}\text { Modèle } 2 \\
\text { Bêta (Signification) }\end{array}$ \\
\hline $\begin{array}{l}\text { Variables } \\
\text { de contrôle : }\end{array}$ & $\begin{array}{l}\text { Sexe } \\
\text { Âge par catégories } \\
\text { Ancienneté professionnelle } \\
\text { Ancienneté fonctionnelle } \\
\text { Ancienneté organisationnelle } \\
\text { Cadres infirmiers } \\
\text { Cadres administratifs } \\
\text { Cadres médecins } \\
\text { Autres cadres }\end{array}$ & $\begin{array}{l}.012(.747) \\
-.046(.342) \\
.039(.380) \\
.008(.867) \\
-.009(.843) \\
.066(.280) \\
.018(.701) \\
-.091(.115) \\
.085(.122)\end{array}$ & $\begin{array}{l}.006(.797) \\
-.034(.310) \\
.025(.405) \\
-.045(.185) \\
-.029(.349) \\
.030(.481) \\
-.007(.832) \\
.030(.485) \\
.033(.383)\end{array}$ \\
\hline $\begin{array}{l}\text { Variables } \\
\text { indépendantes: }\end{array}$ & $\begin{array}{l}\text { Rel_sup } \\
\text { Rel_col } \\
\text { Org_pers_trav } \\
\text { Com_inf_org } \\
\text { Auto_resp } \\
\text { Regl_proc } \\
\text { Dev_pers } \\
\text { Remuneration }\end{array}$ & & $\begin{array}{l}.317(.000)^{* * *} \\
.195(.000)^{* * *} \\
.133(.000)^{* * *} \\
.084(.010)^{*} \\
.114(.001)^{* * *} \\
.038(.207) \\
.077(.025)^{*} \\
.043(.140)\end{array}$ \\
\hline $\begin{array}{l}\text { Récapitulatif } \\
\text { des modèles: }\end{array}$ & $\begin{array}{l}\mathrm{R}^{2}: \\
\text { Variation de } \mathrm{R}^{2}: \\
\mathrm{R}^{2} \text { ajusté : } \\
\text { Statistique F: } \\
\text { Test Durbin-Watson : }\end{array}$ & $\begin{array}{l}.028 \\
.028 \\
.017 \\
2.560(.007)^{* *}\end{array}$ & \begin{tabular}{|l|}
.559 \\
.531 \\
.549 \\
$118.823(.000)^{* * *}$ \\
1.843
\end{tabular} \\
\hline \multicolumn{4}{|c|}{$\mathrm{N}=809$ (seuls les questionnaires complets sont pris en compte) ; $p<.05^{*} / \mathrm{p}<.01^{* *} / \mathrm{p}<.001^{* * *}$} \\
\hline
\end{tabular}

Par contre, si nous nous tournons maintenant vers l'interprétation des résultats du modèle 2, on peut constater que la variance expliquée par toutes nos variables atteint $55.9 \%\left(R^{2}=.559\right)$, et que $53.1 \%$ (variation de $\left.R^{2}=.531\right)$ de cette variance est attribuable uniquement aux variables indépendantes en lien avec les relations sociales et les caractéristiques du travail. Si l'on considère les résultats les plus significatifs, nous pouvons constater, par ordre d'importance, que les relations avec les supérieurs hiérarchiques $(r=.317 ; p<.001)$, les relations avec les collègues $(r=.195 ; p<.001)$, la possibilité d'organiser son travail de manière satisfaisante $(r=.133 ; p<.001)$, l'autonomie et les 
responsabilités $(r=.114 ; p<.01)$, la communication et l'information au sein de l'organisation $(r=.084 ; p<.05)$ et, finalement, les possibilités de développement personnel au sein de l'organisation $(r=.077 ; p<.05)$ sont les variables principales associées significativement et positivement à la satisfaction au travail. Une fois encore, les aspects relationnels et les caractéristiques propres au travail de nos répondants sont les variables clés pour la compréhension de la satisfaction au travail dans le cadre de notre étude.

\section{DISCUSSION}

Les résultats obtenus ci-dessus confirment, en grande partie mais avec quelques surprises néanmoins, nos hypothèses de travail ou attentes théoriques. II paraît clair, au vu des traitements statistiques réalisés, que les aspects relationnels et les caractéristiques du travail de nos répondants sont des variables centrales dans la recherche des antécédents de la satisfaction au travail et du stress, même si dans ce dernier cas l'ensemble de nos variables n'explique qu'environ un tiers de la variance de cette variable dépendante. En conséquence, d'autres facteurs centraux, non identifiés dans cette présente recherche, peuvent avoir des impacts importants à la fois sur le stress mais également sur la satisfaction au travail. Le tableau 5, ci-dessous, récapitule nos différentes hypothèses en les confrontant à nos résultats empiriques.

\section{Tableau 5. Confrontation de nos attentes théoriques avec nos résultats de recherche}

\begin{tabular}{|c|c|c|}
\hline Variables indépendantes & Stress & Satisfaction au travail \\
\hline $\begin{array}{l}\text { Relations avec les supérieurs } \\
\text { hiérarchiques }\end{array}$ & $\begin{array}{c}\text { NS } \\
\begin{array}{c}\text { Hypothèse non supportée } \\
\text { par nos résultats }\end{array} \\
\end{array}$ & $\stackrel{+}{\stackrel{+}{H} \text { Hypothèse supportée }}$ \\
\hline Relations avec les collègues & Hypothèse supportée & $\stackrel{+}{\text { Hypothèse supportée }}$ \\
\hline $\begin{array}{c}\text { Organisation personnelle } \\
\text { du travail }\end{array}$ & Hypothèse supportée & $\begin{array}{c}+ \\
\text { Hypothèse supportée } \\
\end{array}$ \\
\hline $\begin{array}{l}\text { Communication, information } \\
\text { au sein de I'organisation }\end{array}$ & $\begin{array}{c}\text { NS } \\
\text { Hypothèse non supportée }\end{array}$ & $\begin{array}{c}+ \\
\text { Hypothèse supportée }\end{array}$ \\
\hline Autonomie, responsabilités & Hypothèse supportée & 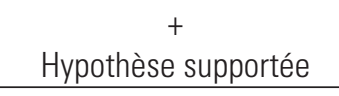 \\
\hline $\begin{array}{l}\text { Règles, procédures et } \\
\text { contraintes administratives }\end{array}$ & $\begin{array}{l}\text { Hypothèse non supportée car nous } \\
\text { attendions une relation inverse }\end{array}$ & $\begin{array}{c}\text { NS } \\
\text { Hypothèse non supportée }\end{array}$ \\
\hline Développement personnel & $\begin{array}{c}\text { NS } \\
\text { Hypothèse non supportée }\end{array}$ & $\stackrel{+}{+}+{ }^{+}$ \\
\hline Rémunération & $\begin{array}{c}\text { NS } \\
\text { Hypothèse non supportée }\end{array}$ & $\begin{array}{c}\text { NS } \\
\text { Hypothèse non supportée }\end{array}$ \\
\hline
\end{tabular}

- = soulignant une association négative avec la variable dépendante

$+=$ soulignant une association positive avec la variable dépendante

$\mathrm{NS}=$ association entre les variables statistiquement non significatives 


\section{, 6.1. Les antécédents du stress}

Si l'on s'intéresse, dans un premier temps, au stress, nous pouvons constater que nos répondants peuvent compter sur un certain nombre de ressources pour y faire face. Aussi, plus les répondants se montrent satisfaits de leurs relations avec leurs collègues de travail et moins ils ont tendance à déclarer un haut niveau de stress. II en va de même pour les variables suivantes : I'organisation personnelle du travail, I'autonomie et les responsabilités et, plus étonnant, les règles, procédures et contraintes administratives. Ce dernier résultat va à l'encontre d'autres conclusions de recherches récentes ayant démontré l'impact négatif des contraintes administratives (ou red tape en anglais) sur la satisfaction et sur le bien-être au travail des agents publics (DeHart-Davis et Pandey, 2005 ; Giauque, Anderfuhren-Biget et al., 2013 ; Giauque, Ritz et al., 2012). Ceci dit, ce résultat plutôt surprenant dans le cadre de notre propre étude, provient probablement de la manière dont nous avons libellé notre item. En effet, il était demandé à notre population de recherche de caractériser, sur une échelle de Likert classique en 5 points ( 1 = très insatisfaisant ; 5 = très satisfaisant), certains aspects de leur travail. Dès lors, plus nos répondants ont déclaré être satisfaits de «l'incidence des procédures et règles à respecter sur le déroulement des activités " et de "l'impact des contraintes administratives " et moins ils ont tendance à exprimer un sentiment de stress important. En d'autres termes, les règles, procédures et contraintes bureaucratiques peuvent également constituer des ressources pour lutter contre le stress si ces dernières facilitent le travail des agents publics. Ceci confirme le fait que les règles, procédures et contraintes bureaucratiques peuvent parfois être habilitantes et donc constituer des ressources importantes pour lutter contre le stress professionnel. En sociologie des organisations, un tel résultat n'est pas très surprenant dans la mesure où des règles formelles, considérées comme légitimes par les acteurs, peuvent contribuer à une meilleure coopération et collaboration (Reynaud, 1997 ; Perrow, 2007). De plus, des règles formelles claires peuvent être également perçues comme stabilisantes dans le cadre d'un contexte organisationnel en pleine transformation, notamment du fait des réformes hospitalières actuelles qui impactent sur le contenu et les pratiques du travail des différents acteurs. En ce sens, précisément, les règles peuvent être considérées comme habilitantes.

Une autre surprise de taille est le fait que les relations avec les supérieurs hiérarchiques ne soient pas une variable associée avec le stress perçu. En termes relationnels, ce qui compte le plus est la relation avec les collègues et non pas la relation avec les supérieurs hiérarchiques, du moins dans la présente recherche. Ce résultat est intéressant à plus d'un titre. II démontre, s'il en était encore besoin, l'importance des liens sociaux de " proximité ", notamment avec le groupe professionnel d'appartenance, lorsqu'il s'agit de faire face au stress. Répétons-le, notre population est spécifique puisqu'elle réunit des cadres intermédiaires qui sont issus d'une corporation professionnelle particulière (la médecine, les soins, la comptabilité, l'intendance, etc.), mais qui ont la lourde tâche 
d'encadrer leurs collègues en se faisant en même temps les représentants de la direction de leur établissement. Dans une telle situation d'entre-deux, il semble bien que les relations avec les collègues constituent une ressource importante pour faire face au stress. En période de réformes managériales et organisationnelles, le groupe professionnel, avec les solidarités qu'implique un tel groupe, constitue une ressource pour faire face aux contraintes. Les relations avec les supérieurs hiérarchiques, qui sont aujourd'hui bien souvent des gestionnaires professionnels, sont probablement moins importantes pour le cadre intermédaire qui souhaite se protéger des nouvelles contraintes émanant des réformes, notamment du fait que ces nouvelles contraintes proviennent précisément des strates hiérarchiques auprès desquelles il doit rendre des comptes. En période de changements, les acteurs ont tendance à resserrer les liens avec les collègues. L'attachement au groupe professionnel reste donc un élément central pour nos répondants afin de maintenir une qualité de vie au travail, résultat confirmant l'importance des identités professionnelles dans le fonctionnement des organisations, notamment publiques et hospitalières (Andersen, 2009 ; Belorgey, 2010 ; Champy, 2009 ; Liaroutzos, 1993).

Autre résultat étonnant : la satisfaction et/ou l'insatisfaction de nos répondants à l'égard de la communication au sein de leur établissement hospitalier ou de la gestion de l'information et des conflits dans leur organisation n'a pas de relation significative avec le stress perçu. La communication/l'information, souvent « fourre-tout » du management, ne constituent donc pas une ressource pour nos acteurs dans le cadre de la présente étude. Finalement, notons que les aspects en lien avec des caractéristiques plus extrinsèques du travail, dont les possibilités de développement personnel (promotion, plan de carrière, formation continue) et la rémunération, ne sont pas associés significativement avec le stress perçu, résultat confirmant le peu d'impact des facteurs extrinsèques du travail sur les attitudes et comportements des salariés (Frey et Jegen, 2001 ; Moynihan, 2010 ; Pfeffer, 2007 ; Taylor, 2008). Relevons également que l'organisation personnelle du travail (les horaires de travail, I'organisation des jours de repos et des vacances, I'équilibre entre vie professionnelle et vie privée, ainsi que la stabilité de l'emploi) constitue une ressource importante pour faire face au stress. Ce dernier résultat fait écho aux conclusions de recherches internationales portant sur d'autres contextes organisationnels (Bakker et Demerouti, 2007 ; Demerouti et al., 2001 ; Johnson et Hall, 1988 ; Schaufeli et Bakker, 2004 ; Siegrist, 1996).

\section{, 6.2. Les antécédents de la satisfaction au travail}

Si l'on s'intéresse maintenant aux antécédents de la satisfaction au travail, nous constatons que la quasi-totalité de nos hypothèses de travail sont supportées par nos résultats empiriques, à l'exception des règles, procédures et contraintes administratives qui ne sont pas significativement associées à notre variable dépendante. Hormis la rémunération également, toutes les autres variables relationnelles et concernant les 
caractéristiques du travail sont corrélées avec la satisfaction au travail dans le sens de nos hypothèses. Peu de surprise, donc, concernant cette partie de notre recherche, mais beaucoup de confirmation quant à l'importance des relations sociales et de certaines caractéristiques du travail en vue de soutenir et développer la satisfaction des agents publics. Nos résultats de recherche ne divergent donc pas significativement des autres enquêtes menées précédemment dans des populations d'agents publics (Barrows et Wesson, 2000 ; Taylor et Westover, 2011 ; Wood et al., 2012). Par contre, cette dernière régression statistique permet de mettre en évidence l'importance des facteurs relationnels et organisationnels dans le développement de cette attitude positive à l'égard du travail. Dans notre cas, pour rappel, plus de $50 \%$ de la variance de la satisfaction au travail est expliquée par nos variables indépendantes, ce qui, en sciences sociales, constitue un résultat particulièrement important. Autrement dit, on peut soutenir l'idée selon laquelle la satisfaction au travail n'est pas avant tout une disposition psychologique individuelle mais plutôt une co-construction entre attentes individuelles à l'égard du travail et les conditions organisationnelles perçues. Ce résultat n'est pas particulièrement novateur, puisque d'éminents spécialistes ont d'ores et déjà fait ce constat précédemment (Demerouti et al., 2001 ; Karasek, 1979), mais il doit être souligné avec force dans un contexte de forte "psychologisation » de la gestion des ressources humaines et des organisations (Brunel, 2008).

\subsection{Des résultats contrastés par catégories de cadres}

La question de savoir si l'appartenance aux différentes catégories de cadres peut avoir un impact différencié en lien avec nos différentes variables indépendantes paraît légitime tant il faut bien admettre des différences " fonctionnelles " et " professionnelles » importantes entre des cadres infirmiers, administratifs ou médecins. Dans les régressions présentées auparavant dans cet article, les différentes catégories de cadres ne semblent avoir aucune relation significative et statistiquement explicative du stress perçu et de la satisfaction au travail. Autrement dit, il existe une certaine convergence entre les différentes catégories de cadres en regard des facteurs de stress et de satisfaction au travail. Pour aller un peu plus loin dans l'analyse, nous nous sommes livrés à des analyses statistiques complémentaires visant à identifier si l'appartenance à une catégorie spécifique de cadres pouvait avoir une relation statistique significative avec nos variables relationnelles et organisationnelles. Et dans ce cadre, des différences en matière de perception apparaissent clairement entre les différentes catégories de cadres. Ainsi, plusieurs constats émergent de nos analyses statistiques complémentaires :

- II existe une relation significative et négative entre la catégorie " cadres médecins " et la variable " relation avec les supérieurs hiérarchiques". Autrement dit, les cadres médecins semblent globalement plus insatisfaits que les autres cadres de leurs relations avec les supérieurs hiérarchiques. Pour les autres catégories 
de cadres, les relations statistiques sont non significatives. Ce premier résultat évoque probablement des tensions ou conflits importants entre les cadres médecins et leurs supérieurs hiérarchiques dans une période de réformes visant à la managérialisation des activités hospitalières. Dès lors, on peut également supposer que les cadres médecins se trouvent dans des situations de conflits/tensions de rôles difficiles à vivre.

- Les catégories " cadres infirmiers", " cadres administratifs " et " autres cadres " sont associées positivement et statistiquement significativement avec la variable " relations avec les collègues ». Dit de manière différente, les cadres infirmiers, administratifs et autres cadres déclarent un degré de satisfaction important concernant leurs relations avec leurs collègues. La relation entre " cadres médecins » et cette dernière variable n'est pas statistiquement significative, suggérant un isolement social plus important pour cette dernière catégorie de cadres.

- Les catégories " cadres médecins " et " cadres infirmiers " sont associées négativement et significativement à la variable " organisation personnelle du travail ». Ce qui implique donc que ces deux catégories de cadres perçoivent plus négativement que les autres les possibilités qu'ils peuvent avoir en termes d'organisation personnelle du travail. II est vrai que dans le contexte de réformes actuelles, les personnels soignants au sens large, se trouvent confrontés à des contraintes importantes qui ne doivent pas faciliter la flexibilité en termes d'horaires de travail, l'organisation des jours de repos et de vacances ou encore la conciliation entre vie profesionnelle et vie privée. Une insatisfaction particulière est ici exprimée par les cadres intermédiaires " soignants", insatisfaction correspondant très probablement à des contraintes spécifiques auxquelles ces cadres doivent faire face, notamment dans des périodes de restructurations organisationnelles.

- Les " cadres médecins " sont également les plus critiques à l'égard de la communication et de l'information au sein de leur organisation. Ils perçoivent plus négativement que les autres catégories de cadres la communication au sein de l'établissement hospitalier, la gestion de l'information et des conflits. Ce résultat est probablement lié au fait qu'ils perçoivent leurs relations avec leurs supérieurs hiérarchiques comme particulièrement insatisfaisantes, laissant présager des conflits importants entre logiques " médicales " et logiques " managériales ". Ce type de résultat confirme des résultats similaires obtenus dans le cadre d'autres recherches, notamment en France (Belorgey, 2010).

- Les "cadres médecins " sont aussi ceux qui déclarent une insatisfaction toute particulière concernant leur autonomie et responsabilité. Ils perçoivent plus négativement que les autres catégories de cadres leur implication dans la prise de décision, leur marge d'autonomie et les responsabilités qui leur sont accordées. 
- Concernant maintenant les règles, procédures et contraintes administratives, les " cadres médecins " et les " cadres infirmiers " les perçoivent comme particulièrement insatisfaisantes. Ces cadres soignants perçoivent donc négativement l'incidence des procédures et règles sur le déroulement des activités, de même que I'impact des contraintes administratives sur leur travail. Les réformes hospitalières actuelles ne sont probablement pas étrangères à ce sentiment négatif à l'égard des règles, procédures et contraintes bureaucratiques. II est clair que l'un des piliers des réformes hospitalières contemporaines visent à mesurer et " contraindre " les actes médicaux et infirmiers en les normalisant, réduisant les actes à des chiffres ou coûts permettant un suivi managérial de l'évolution des dépenses hospitalières. Ces nouvelles normes comptables (Bruno et Didier, 2013), qui sont autant de nouvelles règles du jeu, sont donc plutôt mal vécues par ces acteurs.

Les différents résultats présentés précédemment démontrent bien que les différentes catégories de cadres des hôpitaux dans lesquels nous avons eu la chance d'enquêter ne réagissent pas de la même manière à leur environnement de travail, surtout dans une période de transformations importantes. Les cadres les plus insatisfaits de leurs conditions de travail sont les cadres soignants, principalement les cadres médecins. Ils perçoivent donc des contraintes plus importantes dans leur environnement professionnel que les autres catégories de cadres. Probablement parce que les nouvelles règles du jeu du fonctionnement hospitalier suisse les touchent en priorité. Ne perdons pas de vue que les objectifs d'efficience qui se trouvent à la base des réformes hospitalières passent principalement par une disciplinarisation des personnels médicaux et infirmiers qui doivent se conformer à des forfaits par actes (Diagnostic Related Groups évoqué en début d'article). Dans une telle configuration, il n'est pas étonnant que les cadres médecins et cadres infirmiers soient particulièrement critiques. Ces dernières remarques confirment la thèse, évoquée par certains auteurs, selon laquelle les réformes de type NGP contribuent à une dé-professionnalisation des organisations ou, en tous les cas, visent à s'attaquer à l'autonomie des professionnels (Ferlie et Geraghty, 2007). Cependant, de plus amples investigations en Suisse et ailleurs sont nécessaires afin de confirmer ou infirmer une telle affirmation, à tout le moins dans l'univers hospitalier.

\section{CONCLUSIONS, RECHERCHES FUTURES ET LIMITES}

Notre article vise plusieurs objectifs : identifier les principaux antécédents du stress perçu et de la satisfaction au travail par une démarche quantitative s'appliquant à des données récoltées dans une population de "cadres intermédiaires " exerçant dans des établissements hospitaliers publics de Suisse romande. Si l'étude des déterminants du stress et de la satisfaction au travail n'est pas très novatrice, dans la mesure où il existe une littérature relativement abondante concernant cette thématique, il n'en reste pas moins que notre travail autorise quelques avancées intellectuelles. II vise à combler un 
manque de données statistiques en Suisse permettant de mieux comprendre le phénomène du stress dans les organisations publiques, d'une part, et d'autre part relativement à une population particulière, très peu prise en considération jusqu'à ce jour, à savoir les cadres intermédiaires hospitaliers. Comme la recherche se développe de façon incrémentale, par sédimentation, notre travail contribue à l'édification d'une meilleure connaissance des mécanismes présidant au stress et à la satisfaction au travail dans les établissements hospitaliers suisses romands sans, pour autant, que les résultats présentés ne s'écartent trop des résultats obtenus dans le cadre de recherches internationales. Qui plus est, le fait que cette étude soit menée dans un contexte de réformes n'est pas dénué d'intérêt. Le constat, par exemple, qu'environ $23 \%$ de nos répondants estiment que les changements organisationnels récents vont avoir des impacts défavorables sur la gestion du temps de leurs activités, démontre que les réformes en cours ne sont pas neutres et qu'elles sont susceptibles de remettre en question les principales ressources dont disposent les acteurs pour faire face au stress professionnel. Ils sont également $23.6 \%$ à mentionner que les réformes actuelles vont avoir des impacts négatifs sur leur pouvoir décisionnel, $20 \%$ à déclarer qu'elles auront des effets négatifs sur leur identité professionnelle, $15.9 \%$ à craindre des conséquences négatives sur le travail en équipe et environ $20 \%$ à anticiper des résultats peu propices à la coordination des activités entre collègues. Ces chiffres ne sont pas alarmants, mais étant donné la relative jeunesse des réformes hospitalières, l'on peut s'attendre à des résistances importantes parmi les cadres intermédiaires.

Aussi, si nous avons pu identifier un certain nombre d'antécédents du stress et de la satisfaction, que la plupart d'entre eux concernent des facteurs relationnels et en lien avec les caractéristiques du travail, il apparaît également qu'une proportion importante de nos répondants ne se montrent guère optimistes quant à leurs futures conditions de travail pouvant résulter des réformes en cours. En conséquence, une étude longitudinale, prenant en compte le temps long, la mesure des perceptions sur le plus long terme, pourrait constituer une bonne stratégie pour suivre l'évolution du stress perçu et de la satisfaction au travail dans les établissements publics hospitaliers. Pour notre part, nous ne sommes pas en mesure de donner suite à une telle proposition, mais d'autres recherches pourraient s'en inspirer. Par contre, soulignons le résultat le plus important de notre étude. Le stress perçu et la satisfaction au travail sont avant tout associés à des facteurs relationnels et des caractéristiques du travail ou organisationnelles. Avant d'être des manifestations individuelles, le stress et la satisfaction sont donc des phénomènes sociaux et « structurels ». Bien sûr, il ne serait pas adéquat d'éliminer complètement les origines individuelles du stress et de la satisfaction au travail, certains scientifiques ne s'en privent pas en considérant que le stress est avant tout explicable par des dimensions psychologiques. Psychologisation par ailleurs dénoncée fortement par de nombreux auteurs contemporains qui insistent plutôt sur l'importance 
des " structures " organisationnelles et managériales, elles-mêmes impactant sur les conditions de travail, dans l'explication des phénomènes de souffrance au travail (Clot, 2010 ; de Gaulejac, 2006 ; Thébaud-Mony et Robatel, 2009 ; Vézina, 1999).

La caractéristique synchronique de notre travail constitue donc une première limite dans l'identification des déterminants du stress et de la satisfaction au travail, tant il faut bien reconnaître le caractère contingent de notre enquête. Une deuxième limite a trait à l'utilisation de données statistiques qui concernent avant tout des déclarations d'acteurs. Le caractère déclaratif des enquêtes quantitatives constitue évidemment un biais important, dans la mesure où les réponses au questionnaire peuvent être fonction de l'état d'esprit « du moment ». D'autre part, dans la mesure où nos répondants ont été invités à s'exprimer à la fois sur les déterminants et sur leurs effets dans un même questionnaire, il est fort probable que cette stratégie puisse contribuer à des effets « inflationnistes " de nos résultats (Podsakoff et al., 2003). Relevons également que cette recherche s'intéresse à un groupe d'acteurs, les cadres intermédiaires, qui partagent en commun un certain nombre de caractéristiques et missions organisationnelles mais qui proviennent d'univers professionnels parfois assez éloignés. Cadres médecins, cadres administratifs, cadres infirmiers sont des populations dont l'histoire, le positionnement, les questionnements, les profils, les rôles et les rapports aux autres métiers sont différents. Nous ne rendons probablement pas suffisamment justice à ces différences dans le présent article. D'autres études pourraient ainsi saisir l'opportunité d'étudier ce qui différencie ces différents corps de métier en matière de stress et de satisfaction au travail. Pour notre part, nous avons fait le choix, certes discutable, de relever les antécédents du stress et de la satisfaction qui sont communs aux cadres intermédaires des hôpitaux de Suisse romande. Finalement, soulignons que nous sommes actuellement en train de compléter notre étude par des "focus groups " auprès de notre population de cadres intermédiaires. L'objectif de cette partie " qualitative " de la recherche est de mieux comprendre le sens que nos répondants donnent à leurs déclarations. Pour exemple, savoir que les relations avec les collègues peuvent constituer de précieuses ressources pour affronter le stress ne nous dit pas grand-chose sur le pourquoi de cette déclaration. Que cache donc une telle relation, quels exemples concrets pouvons-nous en donner? Autant de questions qui ne peuvent être valablement appréhendées que dans le cadre d'entretiens. 
Annexe 1. Les variables et items utilisés dans notre questionnaire

\begin{tabular}{|c|c|c|}
\hline Variables & Items et mesures & $\begin{array}{l}\text { Alpha de } \\
\text { Cronbach }\end{array}$ \\
\hline $\begin{array}{l}\text { Variables } \\
\text { de contrôle : }\end{array}$ & $\begin{array}{l}\text { Sexe : femmes = 1; hommes = } 2 \\
\text { Âge par catégories : } \\
\text { - } 1 \text { = } 20 \text { ans et moins } \\
\text { - } 2 \text { = 21-30 ans } \\
\text { - } 3 \text { = } 31-40 \text { ans } \\
\text { - } 4=41-50 \text { ans } \\
\text { - } 5=51-60 \text { ans } \\
\text { - } 6=61 \text { et plus } \\
\text { Ancienneté dans la profession : nombre d'années } \\
\text { Ancienneté dans la fonction : nombre d'années } \\
\text { Ancienneté organisationnelle : nombre d'années }\end{array}$ & \\
\hline $\begin{array}{l}\text { Variables } \\
\text { indépendantes }\end{array}$ & $\begin{array}{l}\text { Toutes les questions sont libellées de la manière suivante: Comment } \\
\text { caractérisez-vous les aspects suivants de votre travail ? (1 = très insatisfaisant ; } \\
5 \text { = très satisfaisant) } \\
\text { Relations avec les supérieurs hiérarchiques - } 4 \text { items (Rel_sup). } \\
\text { - Relations avec vos supérieur-e-s } \\
\text { - Soutien de vos supérieur-e-s dans votre activité } \\
\text { - Reconnaissance de vos supérieur-e-s } \\
\text { - Valorisation des aspects positifs de votre activité par vos supérieur-e-s } \\
\text { Relations avec les collègues - } 4 \text { items (Rel_col) } \\
\text { - Relations avec vos collègues } \\
\text { - Soutien des collègues dans votre activité } \\
\text { - Ambiance de travail } \\
\text { - Possibilité de communiquer ouvertement dans le service } \\
\text { Organisation personnelle du travail - } 4 \text { items (Org_pers_trav) } \\
\text { - Horaires de travail } \\
\text { - Organisation de vos jours de repos et vacances } \\
\text { - Equilibre entre vie professionnelle et vie privée } \\
\text { - Stabilité de l'emploi } \\
\text { Communication et information - } 3 \text { items (Com_inf_org) } \\
\text { - Communication au sein de l'ensemble de votre établissement hospitalier } \\
\text { - Gestion constructive des conflits dans votre établissement } \\
\text { - Gestion de l'information dans votre établissement } \\
\text { Autonomie et responsabilités - } 3 \text { items (Auto_resp) } \\
\text { - Implication dans la prise de décision } \\
\text { - Marge d'autonomie } \\
\text { - Responsabilité accordée } \\
\text { Règles, procédures et contraintes bureaucratiques - } 2 \text { items (Regl_proc) } \\
\text { - Incidence des procédures et règles à respecter sur le déroulement des activités } \\
\text { - Impact des contraintes administratives } \\
\text { Développement personnel - } 3 \text { items (Dev_pers) } \\
\text { - Possibilités de promotion, plan de carrière } \\
\text { - Possibilités de formation } \\
\text { Rémunération }\end{array}$ & .741 \\
\hline
\end{tabular}




\section{BIBLIOGRAPHIE}

AGHO, A. 0., MUELLER, C. W., PRICE, J. L. (1993). « Determinants of Employee Job Satisfaction: An Empirical Test of a Causal Model ». Human Relations, 46(8), 1007-1027.

ANDERSEN, L. B. (2009). « What determines the behaviour and performance of health professionals? Public service motivation, professional norms and/or economic incentives ». International Review of Administrative Sciences, 75(1), 79-97.

BAKKER, A. B., DEMEROUTI, E. (2007). "The Job Demands-Resources model: State of the art ». Journal of Managerial Psychology, 22(3), 309-328.

BAKKER, A. B., VAN EMMERIK, H., VAN RIET, P. (2008). « How job demands, resources, and burnout predict objective performance: A constructive replication ". Anxiety Stress and Coping, 21(3), 309-324.

BARROWS, D., WESSON, T. (2000). " A Comparative Analysis of Job Satisfaction Among Public and Private Sector Professionals ". The Innovation Journal, http://www. innovation.cc/peer-reviewed/job-satisfaction2.htm.

BELORGEY, N. (2010). L'hôpital sous pression. Enquête sur le «nouveau management public». Paris : La Découverte.

BOLGIANI, I. (2002). L'application des nouvelles méthodes de gestion publique dans les secteurs sanitaire et hospitalier : risques et opportunités. Muri : Société suisse pour la politique de la santé (SSPS).

BOZEMAN, B. (2000). Bureaucracy and Red Tape, Upper Saddle River, NJ : Prentice Hall.

BREWER, G. A., WALKER, R. M. (2010). "Explaining Variation in Perceptions of Red Tape: A Professionalism-Marketization Model ». Public Administration, 88(2), 418-438.

BRUCHON-SCHWEITZER, M., DANTZER, R. (1994). Introduction à la psychologie de la santé. Paris : PUF.

BRUNEL, V. (2008). Les managers de l'âme. Le développement personnel en entreprise, nouvelle pratique de pouvoir? Paris : La Découverte.

BRUNO, I., DIDIER, E. (2013). Benchmarking. L'État sous pression statistique. Paris : Editions La Découverte.

BÜSSING, A. (1992). "A dynamic view of job satisfaction in psychiatric nurses in Germany ». Work \& Stress, 6(3), 239-259.

CARPENTIER-ROY, M.-C. (1995). "Santé mentale et travail : avantages et limites de la psychodynamique du travail ». In R. Malenfant, M. Vézina (Eds.), Plaisir et souffrance, dualité de la santé mentale au travail. (Vol. 81) : Groupe de recherche sur les impacts 
sociaux et psychologiques du travail, Centre de santé publique de Québec, ACFAS, Les Cahiers Scientifiques.

CARRICANO, M., POUJOL, F. (2008). Analyse de données avec SPSS. Paris: Pearson Education.

CHAMPY, F. (2009). La sociologie des professions. Paris: PUF.

CLOT, Y. (2010). Le travail à cœur. Pour en finir avec les risques psychosociaux. Paris: La Découverte.

COHEN, D. (2012). Homo Economicus, prophète (égaré) des temps nouveaux. Paris: Albin Michel.

COHEN, S., KAMARCK, T., MERMELSTEIN, R. (1983). « A Global Measure of Perceived Stress ". Journal of Health and Social Behavior, 24(4), 385-396.

DAHOUADI, I., EL AKKREMI, A., IGALENS, J. (2008). « Une analyse critique du management par la qualité totale : implication pour la GRH ». Revue de gestion des ressources humaines, no. 67, 2-22.

DE GAULEJAC, V. (2006). " L'idéologie gestionnaire entre insignifiance et légitimation de l'instrumentalisation ». In M.-D. Perrot, J.-N. DuPasquier, D. Joye, J.-P. Leresche et G. Rist (Eds.), Ordres et désordres de l'esprit gestionnaire. Où vont les métiers de la recherche, du social et de la santé ? (pp. 17-40). Lausanne : Réalités Sociales.

DEHART-DAVIS, L., PANDEY, S. K. (2005). " Red Tape and Public Employees: Does Perceived Rule Dysfunction Alienate Managers? ॥. Journal of Public Administration Research and Theory, 15(1), 133-148.

DEJOURS, C. (1987). " Souffrance et plaisir au travail : I'approche de la psychopathologie du travail ». In C. Dejours (Ed.), Plaisir et souffrance dans le travail, tome 1. Paris : CNRS.

DEMEROUTI, E., BAKKER, A. B., NACHREINER, F., SCHAUFELI, W. B. (2001). « The Job Demands-Resources model of burnout ». Journal of Applied Psychology, 86 (3), 499-512.

DEMMKE, C., MOILANEN, T. (2010). Civil Service in the EU of 27: Reform Outcomes and the Future of the Civil Service. Berlin: Peter Lang.

EMERY, Y., GIAUQUE, D. (Eds.). (2007). Dilemmes de la GRH publique. Lausanne: LEP.

EVETTS, J. (2013). "Professionalism: Value and ideology ". Current Sociology, doi:10.1177/0011392113479316.

EVETTS, J. (2009). "New Professionalism and New Public Management: Changes, Continuities and Consequences ". Comparative Sociology, 8(2), 247-266.

FELDMAN, M. S., MARCH, J. G. (1991). " L'information dans les organisations: un signal et un symbole ». In J. G. March (Ed.), Décisions et organisations (pp. 255-275). Paris, Editions d'Organisation. 
FERLIE, E. B., SHORTELL, S. M. (2001). " Improving the Quality of Health Care in the United Kingdom and the United States: A Framework for Change ". The Milbank Quaterly, 79(2), 281-315.

FERLIE, E., ASHBURNER, L., FITZGERALD, L., PETTIGREW, A. (Eds.). (1996). The New Public Management in Action. Oxford: Oxford University Press.

FERLIE, E., GERAGHTY, K. J. (2007). "Professionals in Public Services Organizations. Implications for Public Sector "reforming" ». In E. Ferlie, L. E. Lynn, C. Pollitt (Eds.), The Oxford Handbook of Public Management (pp. 422-445). Oxford: Oxford University Press.

FREY, B. S. (1997). Not Just for the Money: An Economic Theory of Personnel Motivation. Cheltenham Brookfield: Edward Elgar Publishing.

FREY, B. S., JEGEN, R. (2001). " Motivation crowding theory ". Journal of Economic Surveys, 15(1), 589-611.

GIAUQUe, D., ANDERFuhren-Biget, S., VARONE, F. (2013). " Stress Perception in Public Organisations: Expanding the Job Demands-Job Resources Model by Including Public Service Motivation ». Review of Public Personnel Administration, 33(1), 58-83.

GIAUQUE, D., RITZ, A., VARONE, F., ANDERFUHREN-BIGET, S. (2012). " Resigned but Satisfied: The Negative Impact of Public Service Motivation and Red Tape on Work Satisfaction ». Public Administration, 90(1), 175-193.

HAYDUK, L. (1987). Structural equation modeling with LISREL: Essentials and advances (Vol. 13). Baltimore: John Hopkins University Press.

HUFTY, M. (Ed.). (1998). La pensée comptable. État, néolibéralisme, nouvelle gestion publique. Paris/Genève: PUF/Les Nouveaux Cahiers de I'IUED.

JOHNSON, J. V. (1986). The impact of workplace social support, job demands and work control upon cardiovascular disease in Sweden. Unpublished doctoral dissertation, Johns Hopkins University.

JOHNSON, J. V., HALL, E. (1988). " Job strain, work place, social support, and cardiovascular disease ». American Journal of Public Health, 78(10), 1336-1342.

JUDGE, T. A., CHURCH, A. H. (2000). " Job Satisfaction: Research and Practice ". In C. L. Cooper \& E. A. Locke (Eds.), Industrial and Organizational Psychology: Linking Theory with Practice (pp. 166-198). Oxford: Blackwell.

JUDGE, T. A., KLINGER, R. (2007). " Job Satisfaction. Subjective Well-Being at Work ». In M. Eid \& R. Larsen (Eds.), The Science of Subjective Well-Being (pp. 393-413). New York: Guilford Publications.

KAHN, R. L., WOLFE, D. M., QUINN, R. P., SNOEK, J. D. (1964). Organizational stress: Studies in role conflict and ambiguity. New York: Wiley. 
KARASEK, R. (1979). " Job demands, job decision latitude, and mental strain: implications for job redesign ". Administrative Science Quarterly, 24(2), 285-308.

LIAROUTZOS, 0. (1993). L'identité professionnelle des agents administratifs. Université Paris VIII Vincennes, St-Denis.

LOCKE, E. A. (1976). "The Nature and Causes of Job Satisfaction ». In M. D. Dunnette (Ed.), Handbook of Industrial and Organizational Psychology (pp. 901-969). Chicago, IL: Rand McNally.

MASLACH, C., JACKSON, S. E., LEITER, M. (1996). Maslach Burnout Inventory: Manual (3rd ed.). Palo Alto, CA: Consulting Psychologists Press.

MAUGERI, S. (2006). Au nom du client : management néo-libéral et dispositifs de gestion. Paris: L'Harmattan.

MINTZBERG, H. (1982). Structure et dynamique des organisations. Paris: Les Editions d'Organisation.

MOWDAY, R. T., PORTER, L. W., STEERS, R. M. (1982). Employee-Organization Linkages: The Psychology of Commitment, Absenteeism, and Turnover. New York: Academic Press.

MOYNIHAN, D. P. (2010). " A workforce of cynics? The effects of contemporary reforms on public service motivation ». International Public Management Journal, 13(1), 24-34.

NOBLET, A., RODWELL, J., MCWILLIAMS, J. (2006). "Organizational Change in the Public Sector: Augmenting the Demand Control Model to Predict Employee Outcomes under New Public Management ». Work \& Stress, 20(4), 335-352.

NOBLET, A. J., RODWELL, J. J. (2009). « Identifying the Predictors of Employee Health and Satisfaction in an NPM Environment ». Public Management Review, 11(5), 663-683.

PANDEY, S. K., SCOTT, P. G. (2002). « Red tape: A review and assessment of concepts and measures ". Journal of Public Administration Research and Theory, 12(4), 553-580.

PAPART, J.-P. (2002). Le raisonnement scientifique appliqué au domaine de la santé. Lausanne: Editions Réalités Sociales.

PAPART, J.-P. (2007). Lien social et santé mentale. Genève: Editions Médecine et Hygiène.

PERROW, C. (2007). "Why Bureaucracy? ». In A. S. Wharton (Ed.), The Sociology of Organizations (pp. 23-51). Los Angeles: Roxbury.

PESQUEUX, Y. (2008). Qualité et management. Une analyse critique. Paris: Economica.

PFEFFER, J. (2007). " Human resources from an organizational behavior perspective: Some paradoxes explained ». Journal of Economic Perspectives, 21(4), 115-134. 
PODSAKOFF, P. M., MACKENZIE, S. B., JEONG-YEON, L., PODSAKOFF, N. P. (2003). "Common Method Biases in Behavioral Research: A Critical Review of the Literature and Recommended Remedies ". Journal of Applied Psychology, 88(5), 879.

REYNAUD, J.-D. (1997). Les règles du jeu. L'action collective et la régulation sociale. Paris: Armand Colin.

RICHARDSEN, A. M., BURKE, R. J., LEITER, M. P. (1992). " Occupational Demands, Psychological Burnout and Anxiety among Hospital Personnel in Norway ". Anxiety Stress and Coping, 5(1), 55-68.

SCHAUFELI, W., ENZMANN, D. (1998). The burnout companion to study and practice: A critical analysis. New York: Taylor \& Francis.

SCHAUFELI, W. B., BAKKER, A. B. (2004). « Job demands, job resources, and their relationship with burnout and engagement: a multi-sample study ". Journal of Organizational Behavior, 25(3), 293-315.

SIEGRIST, J. (1996). "Adverse health effects of high effort low-reward conditions". Journal of Occupational Health Psychology, 1(1), 27-41.

SIEGRIST, J., PETER, R., JUNDE, A., CREMER, P., SIEDEL, D. (1990). " Low status control, high effort at work and ischemic heart disease: prospective evidence from blue-collar men ». Social Science \& Medicine, 31(10), 1127-1134.

SIEGRIST, J., SIEGRIST, K., WEBER, I. (1986). « Sociological concepts in the etiology of chronic disease: the case of ischemic heart disease ". Social Science \& Medicine, 22(2), 247-253.

STAINES, A. (2000). « Benefits of an ISO 9001 certification : the case of a Swiss regional hospital ». International Journal of Health Care Quality Assurance, 13(1), 27-33.

STAINES, A. (2007). La relation "programme qualité» / "résultats cliniques". Université Jean Moulin, Lyon 3, Lyon.

STAW, B. W. (1984). "Organizational behavior: A review and reformulation of the field's outcome variables ". Annual Review of Psychology, 35(1), 627-666.

STEIJN, B. (2004). « Human Resource Management and Job Satisfaction in the Dutch Public Sector ». Review of Public Personnel Administration, 24(1), 291-303.

SZILAGYI, A. D., WALLACE, M. J. (1983). Organizational behavior and performance. Glenview, IL: Scott \& Co.

TAYLOR, J. (2007). « The impact of public service motives on work outcomes in Australia: A comparative multi-dimensional analysis ». Public Administration, 85(4), 931-959.

TAYLOR, J. (2008). " Organizational Influences, Public Service Motivation and Work Outcomes: An Australian Study ». International Public Management Journal, 11(1), 67-88. 
TAYLOR, J., WESTOVER, J. H. (2011). « Job Satisfaction in The Public Service ». Public Management Review, 13(5), 731-751.

THÉBAUD-MONY, A., ROBATEL, N. (Eds.). (2009). Stress et risques psychosociaux au travail. Paris: La Documentation française.

THELEN, L. (2007). La justice dans les organisations. Des discours aux pratiques. Zurich: Jobindex media ag.

VAN DEN BROECK, A., VANSTEENKISTE, M., DE WITTE, H., LENS, W. (2008). " Explaining the relationships between job characteristics, burnout, and engagement: The role of basic psychological need satisfaction ». Work and Stress, 22(3), 277-294.

VARONE, F., DE VISSCHER, C. (2004). " La nouvelle gestion publique "en action» " [Introduction d'un numéro de la revue et présentation synthétique des articles de la revue]. Revue Internationale de politique comparée, 11(2), 177-185.

VÉZINA, M. (1999). "Stress et psychodynamique du travail : de nouvelles convergences ". Travailler. Revue Internationale de Psychopathologie et de Psychodynamique du Travail, 1(2), 201-218.

WEAVER, C. V. (1980). " Job satisfaction in the United States in the 1970s ". Journal of Applied Psychology, 65(3), 364-367.

WOOD, S., VAN VELDHOVEN, M., CROON, M., DE MENEZES, L. M. (2012). « Enriched job design, high involvement management and organizational performance: The mediating roles of job satisfaction and well-being ". Human Relations, online published (doi: 10.1177/0018726711432476).

YOUSEF, D. (2000). " Organizational Commitment and Job Satisfaction as Predictors of Attitudes toward Organizational Change in a Non-Western Setting ». Personnel Review, 29(5), 567-592. 